\title{
Important Mycotoxins, Iran Status
}

\author{
Mansoureh Mirabolfathy ${ }^{1}$, Rouhollah Karami Osboo ${ }^{1 *}$ and Vahid Rahjoo ${ }^{2}$ \\ ${ }^{1}$ Mycotoxins Research Laboratory,Iranian Research Institute of Plant Protection, Agricultural Research Education and Extension Organization(AREEO), \\ Tehran, Iran \\ ${ }^{2}$ Seed and Plant Improvement Institute (SPII), Agricultural Research, Education and Extension Organization (AREEO), Karaj 31585, Iran
}

Submission: June 10, 2019; Published: July 10, 2019

*Corresponding author: Rouhollah Karami Osboo, Mycotoxins Research Laboratory, Iranian Research Institute of Plant Protection, Agricultural Research Education and Extension Organization (AREEO),Tehran, Iran

\section{Abstract}

A review of reports on mycotoxins demonstrates that aflatoxins, fumonisins, and deoxynivalenol are the most important while zearalenone and ochratoxin also were found frequently in Iran. Natural mycotoxin contaminations of the most important plant grains and commodities have been reported during the recent decade. In 2013 more than $90 \%$ of wheat samples collected at harvest time in Ardabil province was contaminated to deoxynivalenol, with the average amount of $1.7 \mu \mathrm{gg}^{-1}$. Wheat, barley, maize, and rice were tested for zearalenone contamination but generally, contaminations were below than $200 \mu \mathrm{gkg}^{-1}$. Fumonisin contamination was reported in many research and maize is one of the most contaminated cereals to fumonisin, in some maize samples $10 \mu \mathrm{gg}^{-1}$ of this carcinogenic compound was detected. Aflatoxins, the most thoroughly studied and the best understood of mycotoxins have occurred in foodstuffs commonly all over the world. Tree nuts, particularly pistachio nuts, are highly susceptible to contamination by aflatoxins, $32 \%$ of the 122 isolates of $A$. flavus isolates from pistachios, produce more than $2 \mu g k g-1$ aflatoxins, also aflatoxin contamination also was detected in fig, almond, and hazelnut. Cereals could also be contaminated to aflatoxins and sometimes $100 \%$ of samples were contaminated to aflatoxins. AFB1 was detected in $43.6 \%$ of maize samples, in which only $22.5 \%$ were contaminated to higher than MRL level. Barley, grape, raisin, and coffee are the major commodity that could contaminate to ochratoxin A; heavy contamination of raisin was reported in damaged raisin sample in Iran. There are a few works on herbal medicine contamination to mycotoxin and natural occurrence of AFs in herbal medicines was less than tolerance level. Contaminations of the most important mycotoxins in food have been reviewed in this article.

Keywords: Important mycotoxins; Iran; Nuts; Cereal; Dairy food; Management

\section{Introduction}

Mycotoxin contamination of food occurs as a result of crop invasion by some fungi, including Fusarium spp., Penicillium spp. and Aspergillus spp. These fungi produce highly toxic secondary metabolites, the so-called mycotoxins. They can be very harmful to man and animal. In 1960 the Turkey "X" disease was caused by a mycotoxin (aflatoxin) produced by molds belonging to the Aspergillus flavus group. It resulted in the death of 100,000 turkeys in the United Kingdom. Here with the search for mycotoxins began. Today, approximately 700 mycotoxins are known, having different negative effects on one or several organs in the human or animal body [1]. Consumption of undesirable levels of mycotoxins such as trichothecenes, fumonisins, zearalenone, and aflatoxins has carcinogenic, mutagenic, teratogenic and estrogenic effects for human and animal health. Different kinds of mycotoxins have been identified and reported on a wide range of host plants especially cereals and nut including wheat, rice, barley, pistachio, almond etc. which are Iran's major crops. Several investigations have been done on mycotoxin contamination levels of wheat, maize, rice, and barley during the past decade in Iran.

\section{Mycotoxins in wheat and barley}

Wheat (Triticum aestivum L.) and barley (Hordeum vulgare L.) are the most important crops in Iran. The cultivation of wheat and barley in Iran goes back to the origin of agriculture itself. Based on
Ministry of Jihad-e-Agriculture of Iran [2] statistics, Iran wheat's production was about $12400000 \mathrm{t}$, in 2016, and barley's production was about 2974039 t. Both wheat and barley are susceptible to Fusarium head blight (FHB) disease caused by members of the Fusarium graminearum complex group [3]. These fungi infect the crop in wet and cool weather during flowering and grain filling stages and produce mycotoxins that accumulate in the developing kernels. The most-often reported mycotoxins in raw agricultural commodities (after aflatoxins), are those produced by various Fusarium species. In all the world grain contamination by Deoxynivalenol (DON) and zearalenone (ZEA) frequently is encountered (Figure 1).

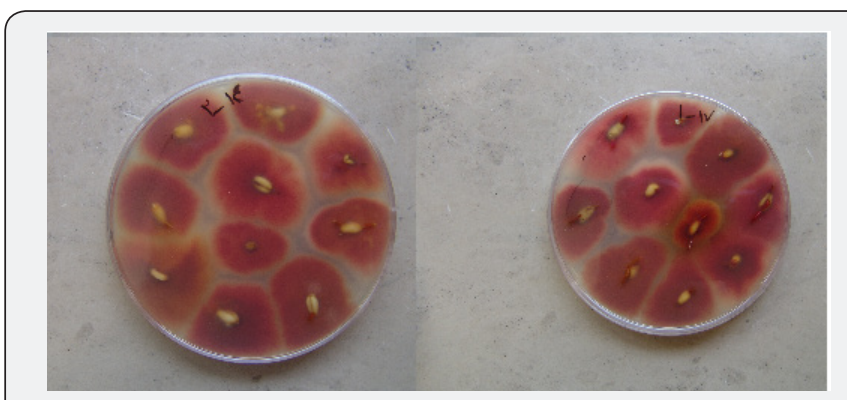

Figure 1: Colony growth of Fusarium graminearum from natural wheat contamination. 
DON is a type B trichothecene mycotoxin produced by several Fusarium species, especially the $F$ graminearum complex group. The synthesis of protein and DNA is inhibited by trichothecenes and many of their toxic effects stem from this mechanism. General signs of trichothecene toxicity in animals include weight loss, decreased feed conversion, feed refusal, vomiting, bloody diarrhea, severe dermatitis, hemorrhage, decreased egg production, abortion, and death. When the advisory levels were issued, there was great concern about the safety of this sort of wheat contamination, which might be used for human food as well as animal feed. The maximum limit level for DON, ZEA, OTA, and AFs in wheat is $1000,200,5$, and $15 \mathrm{ng} / \mathrm{g}$ and in barley is 1000,400 , 50, and 50ng/g, respectively [4]. Mirablolfathy et al. [5] surveyed DON contamination of 227 wheat samples from Golestan, Ardabil, Azarbaijan, and 154 barley samples from Golestan province all located in the north and northwest of Iran, each 10-20kg sampled was ground and sub sampled by Romer mill, all samples collected through different stages including pre-harvest, harvest, enter to silo and exit from silo produced in 2006. Competitive enzymelinked immunosorbent assay and HPLC+ IAC methods were used for evaluating the natural mycotoxin contaminations in that survey. DON was detected in $44.9 \%$ of the wheat samples collected from Golestan province at levels from 18.53 to $192.81 \mathrm{ng}$ $\mathrm{g}^{-1}$, with an average level of 40.99ng g-1. DON was not found in wheat samples of West Azarbaijan and only one sample from East Azarbaiejan was contaminated in this year. DON contamination of wheat and barley produced at north and northwest of Iran in 2006 was much lower than the world regulatory limit for cereals. US Food and Drug Administration advisory level for DON in wheat/ derived products like flour, bran, and germ that may be consumed by a human is $1 \mu \mathrm{gng}^{-1}$. So, the contamination level of Golestan wheat and barley product was lower than the advisory level for deoxynivalenol.

The severe epidemics of Fusarium head blight (FHB) as a devastating disease of cereal crops has occurred on wheat in North and Northwest Iran. Following the outbreak and heavy losses of wheat scab disease at Ardabil province in 2011, forty samples of wheat were collected from Parsabad and Bilesavar at Moqan area (Ardabil province) at harvest time and five samples after three months storing in local storages. Natural contamination of wheat samples to DON was determined by using HPLC method [6], DON was detected in $90 \%$ of samples collected at harvest time ranged from 1.06 to $2.06 \mu \mathrm{gg}^{-1}$, with the average amount of $1.7 \mu \mathrm{gg}^{-1}$. Mean of the incidence of F. graminearum was $70.91 \%$ in irrigated system cultivars. DON potential production of the $29 \mathrm{~F}$ graminearum isolates obtained from wheat samples of this area ranged from 17.82 to $2397.33 \mathrm{ngg}^{-1}$. Levels of DON in the samples collected after two months storing ranged from 3.08 to $7.49 \mu g^{-1}$. In Iran advisory level for DON in wheat derived products are consumed by a human is $1 \mu \mathrm{g} / \mathrm{kg}$. Population analysis of the F graminearum species complex isolated from the wheat crop of this area was considered as the purpose of the study of Mianabi et al. [7]. During Jun till July of 2011, the FHB wheat contaminated samples were collected from wheat fields and harvested wheat crop through Ardabil area. The 321 F graminearum isolates were identified based on morphological characters. The identification of all 321 isolates was confirmed molecularly using Nicholson's specific $F$. graminearum primers. The results of PCR products showed that $90 \%$ of $F$. graminearum isolates (287 out of 321 isolates) were $\mathrm{F}$. asiaticum while in $10 \%$ of isolates were not $F$. asiaticum but they were in the clade of $F$ graminearum. The results showed that F. asiaticum was the predominant species causing Fusarium head blight (FHB) of wheat resulting in DON production throughout all regions of Ardabil province. Sixty isolates of F. graminearum obtained from infected wheat farms of Iran were tested for the ability of DON, nivalenol, and acetylene derivations production using HPLC-SPE method [8]. The results showed that among representative tested isolates, $77 \%$ produced $15-$ AcDON and $46 \%$ produced NIV. Also, none of the DON-producing isolates was able to produce NIV. Whereas the low levels of DON (10- 90ngg-1) were observed in F. graminearum isolates producing high levels of NIV. In another study, the in vitro production of DON was evaluated in 41 Fusarium isolates collected from wheat kernels associated with the scab disease, in Moghan area/Northwest Iran [9]. According to morphological characteristics and using valid descriptions, all isolates belonged to $F$ graminearum species complex and $F$. culmorum of which the former was dominant, results indicated that $54.5 \%$ of studied isolates produced DON but there was no significant relationship between this property and cultivars or subregions or years. The maximum content of DON was detected in $F$. graminearum isolates. The highest level of DON was produced by the F. graminearum isolate from cv. Izen green growth in Moghan Agro-industry company fields in 2014 at the rate of $5827.11 \mu \mathrm{gkg}^{-1}$.

DON contamination of 153 barley samples 2004 to 2005 in Golestan, collected through different stages including pre harvest, harvest, enter to silo and exit from silo were determined by competitive enzyme immunoassay method for the quantitative analysis of deoxynivalenol. Seventy percent of barley samples were contaminated to DON with the range of $15.19-280.6 \mu \mathrm{gkg}$ ${ }^{1}$ and the average amount of contamination was $58.54 \mu^{2} \mathrm{gg}^{-1}[10]$.

ZEA and its derivatives are oestrogenic mycotoxins produced only by Fusarium spp., primarily by $F$ graminearum and $F$. culmorum. ZEA is a b-resorcylic acid lactone that can bind, albeit poorly, to oestrogen receptors and stimulate female sex hormone responses. Therefore, feedstuffs containing ZEA may cause problems such as hyperoestrogenism in raising livestock. Swine are the most sensitive large animal species, but ZEA also causes problems in dairy cattle, chickens and turkeys. Adverse effects have been observed at ZEA concentrations as low as $1.8 \mu \mathrm{g} \mathrm{g}^{-1}$, but they are usually observed at $10-20 \mu \mathrm{g} \mathrm{g}^{-1}$. Generally, ZEA concentrations are well below $1 \mathrm{mgkg}^{-1}$ (the level in the feed that can cause oestrus in swine) in processed cereal foods, but higher amounts can be encountered in some feeds. The maximum tolerance level of ZEA in different countries varies from 30 to $1000 \mu \mathrm{gkg}^{-1}$ and is $200 \mu \mathrm{gkg}$ ${ }^{1}$ in Iran. 
Based on the results obtained from a survey [11] on wheat samples collected from Golestan province, the mean level of ZEA contaminated samples were $72 \mu \mathrm{gkg}^{-1}$ and the range of contamination was $39-104 \mu \mathrm{gkg}^{-1}$.

Aflatoxins (AFs), the most thoroughly studied and best understood of the mycotoxins, are a group of structurally similar polysubstituted coumarins. These extremely toxic metabolites are produced by common molds, Aspergillus flavus, Aspergillus parasiticus, and Aspergillus nomius. There are 4 majors naturally occurring AFs, the most hepatotoxic being aflatoxin B1 (AFB1), and 3 structurally similar compounds, aflatoxins B2, G1, and G2. The principal biological effects of AFs are carcinogenicity, immunosuppression, mutagenicity, and teratogenicity. Aflatoxins cause liver damage; decrease milk yield, egg production, and overall performance; and suppress immunity in animals after the consumption of low dietary concentrations of aflatoxins. The occurrence of AFs in foodstuffs is common all over the world. Wheat samples from Mazandaran, Guilan, Zanjan, Kermanshah, and Khuzestan, in different places, the average contamination and relatively deviation of samples from the 14 cities, were $8.32 \mu \mathrm{gkg}$ ${ }^{1}$ and $3.78 \mu \mathrm{gkg}^{-1}$, respectively; indicate the presence of AFs in newly harvested domestic wheat crops [12]. Beheshti et al. [13], reported that between 60 barley samples, 5 samples and 2 of 22 wheat samples were contaminated to AFB1, but same as Babaei's research, the concentrations in contaminated samples were below than the maximum AFB1 level of Iranian and EU regulations, both being $5 \mu \mathrm{gkg}^{-1}[4]$.

The presence of ochratoxin (OTA) has been determined in barley, malt and beer samples; during the post-harvest stage, fungal infection of barley affects the amount of OTA in beverages [6]. Fourteen barley samples from Golestan and Mazandaran provinces in the north of Iran (destined for animal feed) were analyzed for OTA and no contamination was observed [14].

\section{Mycotoxins in rice}

Iranians consume 3.2 million tons of rice a year, of which more than 2.2 million tons are supplied by domestic farmers. The average per capita consumption of rice in Iran is $45.5 \mathrm{~kg}$, which makes Iranians the $13^{\text {th }}$ biggest rice consumer. There are many reports of rice contamination to AFs in all over the world and Iran. The maximum AFB1 level of Iranian regulation is being $10 \mu \mathrm{gkg}^{-1}$ and Iran National Standard Organization (INSO) has set the maximum limit for OTA levels in human and animal feed and rice at 5 ngg $^{-1}$ [4]. 80 rice samples (62 imported and 18 Iranian) were randomly collected in 2014 and their contamination with aflatoxin B1, B2, and G1, OTA and ZEA were determined, OTA and ZEA were found in none of the samples. But, $54.8 \%$ of samples of imported rice and $22.2 \%$ samples of Iranian rice were contaminated with AFs [15]. Among the 275 imported rice samples analyzed, aflatoxin B1 and B2 were detected in 211 samples (76.72\% of total) [16]. Feizy [5] and coworker analyzed a total of 261 rice samples, that $68.9 \%$ of them contained aflatoxin B1 at levels greater than $0.2 \mathrm{ngg}^{-1}$ [5].
AF contamination of all 45 rice samples collected from Yasuj area was lower than the national standard in Iran. The average amount of total AF in types such as Gerdeh, Champa, Shamim, and Fajr, is $6.53,6.14,4.54$ and 6.12 ngg $^{-1}$, respectively [17].

One hundred and twenty (120) samples of 8 kinds of rice purchased from retail markets in Shahrekord, Iran from January to September 2010 were surveyed for the presence of ochratoxin (OTA) using ELISA method [18]. Analytical results showed a frequency of contamination of $20.8 \%$ of the total analyzed rice samples. Levels of OTA in positive samples ranged between 1.07 and 20. 83ng/g. Out of 120 total samples, 3.3\% showed OTA contamination above the permissible limits of $5(\mathrm{ng} / \mathrm{g})$. Seasonal evaluation of the data indicated a significant difference in contamination levels $(\mathrm{p}<0.05)$. There was no OTA in $47(21.36 \%)$ rice samples out of 220 samples, while in the 173 (78.64\%) remaining samples, OTA was detected at $4.54 \mathrm{ngg}^{-1}$ average level [19]. OTA and AFB1 of 10 domestic and imported rice samples collected from Mazandaran were evaluated using AA-DLLMEHPLC FLD method. OTA average level was 3.87 and $3.15 \mathrm{ngg}^{-1}$ respectively in domestic and imported rice samples, but none were contaminated to AFs [20].

\section{Mycotoxins in maize and sorghum}

Maize (Zea mays L.) is one of the major crops in Iran with the production of approximately 1.65 million tons per year [2]. Based on USDA estimation maize harvested area in Iran is about 210,000 HA. Mycotoxin contamination in maize grain is a worldwide threat to both safeties of human food and animal feed [13]. The maximum limit for DON, ZEA, OTA, fumonisins (FUM) and AFs levels in maize was $1000,200,50,1000$ and $30 \mathrm{ngg}^{-1}$ [4].

Two of the most problematic mycotoxins in maize are AFs produced by the fungi Aspergillus flavus and A. parasiticus [21], and fumonisins produced predominantly by the fungus Fusarium verticillioides (Sacc.) Nirenberg (syn. F. moniliforme) (Figure 2 \& 3) [22].

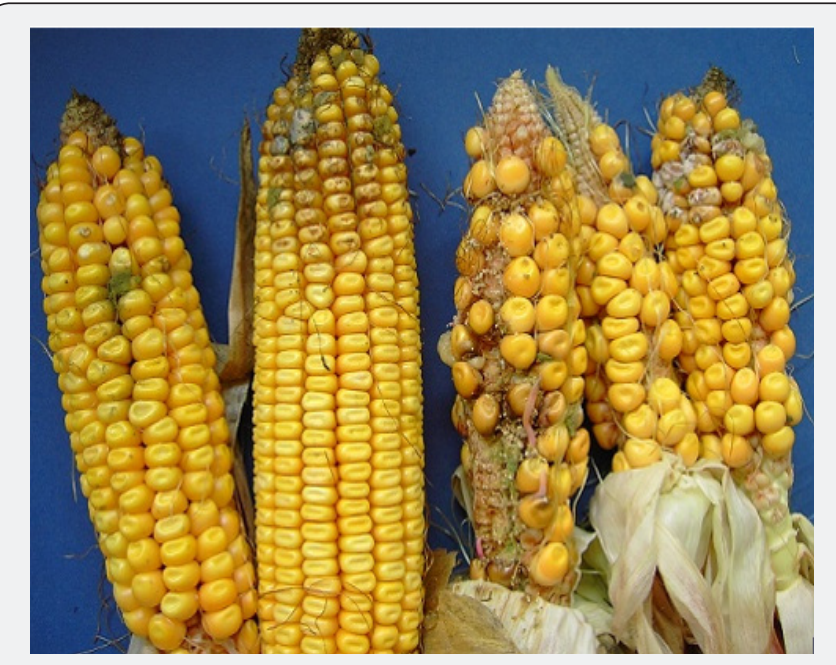

Figure 2: Natural contaminated corn ear to Aspergillus flavus. 


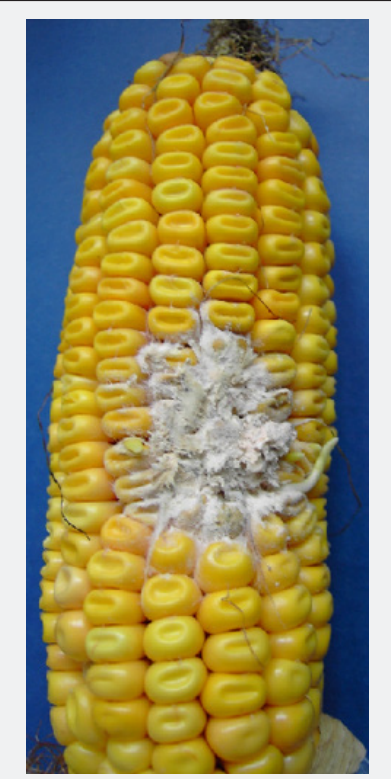

Figure 3: Natural contaminated corn ear to Fusarium proliferatum.

AFB1 contaminations investigated in 373 maize kernel samples by Karami-Osboo et al. [23] collected during 2006-2008 at harvest stage, from different agro-climatic regions of the major maize production area of Iran, including Ardabil (North West), Khuzestan (South West) and Fars at the South of Iran. AFB1 was determined by CD-ELISA and was detected in 146 samples (43.6\%), in which only $22.5 \%$ were contaminated to higher than MRL level. The number of AFs in maize samples varied across the years, the percentage of contamination in 2006, 2007 and 2008 were $86.7 \%, 71.4 \%$, and $100 \%$ respectively for Ardabil, 63.4\%, $41.3 \%$ and $24.5 \%$ for Khuzestan, $17 \%, 14.8 \%$ and $27.6 \%$ for Fars province. The mean of contamination was $15.63,57.67$ and $154.13{\mu g^{-1}}^{-1}$ for Ardabil; $14.41,35.93$ and $1.61 \mu g^{-1}$ for Khuzestan; $0.9,2.12$ and $36.39 \mathrm{mg} / \mathrm{kg}$ for Fars province. The most contaminated maize samples belonged to Ardabil and Khozestan provinces with dry land and high temperatures environmental conditions and drought stresses during the studied years. Aspergillus flavus was isolated more frequently than A. parasiticus and the isolates with numerous small sclerotia produced a greater number of AFs. Fumonisins are a group of mycotoxins produced by Fusarium verticillioides (Sacc.) Nirenberg

Studies about fumonisins in Iran [14,24-28] indicate that fumonisin B1 is the important mycotoxin presents in maize kernels before harvesting and during storage procedures.

Fumonisin B1 (FB1) is the most abundant member of the group. It has been shown to be hepatocarcinogenic and nephrocarcinogenic in male rats and hepatocarcinogenic in female mice. These toxins have been associated with high incidences of esophageal cancer in some countries. Ghiasian et al., [26] analyzed all maize samples collected in 2000 from Fars, Kermanshah, Khuzestan, and Mazandaran. All samples from Mazandaran were contaminated with fumonisins with a mean level of total fumonisins of $10.7 \mu \mathrm{gg}^{-1}$. In contrast, the incidence of fumonisin contamination

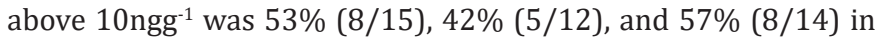
the samples from Fars, Kermanshah, and Khuzestan, respectively, and the corresponding mean total fumonisin levels were 215, 71, and 174 ngg $^{-1}$, respectively, which were significantly lower than the fumonisin contamination in samples from Mazandaran. Mirabolfathy et al. [27] evaluated the amount of fumonisin B1 in 46 maize samples which were collected at pre-harvest, harvest and post-harvest stages from Golestan province in the northern region of Iran. All samples were contaminated with fumonisin B1, the range of contamination varied 0.261 to $6.9 \mu \mathrm{g} \mathrm{g}^{-1}$ and the mean of contamination was $2.6 \mu \mathrm{gg}^{-1}$. There was no significant difference in the contamination of samples at different sampling stages.

Rahjoo [40] and coworker designated research to study on fumonisin accumulation in different grain sorghum and maize genotypes in Iran. To do this, they evaluated different maize and sorghum genotypes based on an RCBD with three replications at four locations over two years. The results of maize experiments demonstrated that genotypes 2 and 1 with $40.9 \%$ and 13\% DS respectively were the most susceptible and resistant genotypes respectively. Also genotypes 2 and 1 had the highest and the least level of fumonisins with 72.6 and $8.9 \mu \mathrm{g} \mathrm{g}^{-1}$ respectively in comparison to the other maize genotypes. There was an appropriate correlation between fumonisin contamination and disease severity measured on different maize genotypes $(\mathrm{r}=+0.93, \mathrm{P}<0.01)$. The results of sorghum experiments at all locations for two years demonstrated that genotypes 1 and 7 with $42.8 \%$ and $27.2 \%$ DS respectively were the most susceptible resistant genotype respectively. Fumonisin production measured of all genotypes was not more than $2 \mu \mathrm{gg}^{-1}$ and there was a low correlation between fumonisin contamination and disease severity measured on different sorghum genotypes. The results of this experiment demonstrated that fumonisin production level in maize kernels (may be as maize kernels are the main host of this fungus) was significantly higher than sorghum kernels. This is the first report of fumonisin analysis of sorghum hybrids in Iran.

In order to determine fumonisins production on different grain sorghum genotypes, Rahjoo and Atabaki [41] carried out a field trial based on a randomized complete block design with 10 treatments and three replications at four locations: Karaj, Gorgan, Moghan and Sari stations in 2011. They inoculated the sorghum panicles by spraying of spore suspension isolates at flowering stage. The results of fumonisins analysis obtained from the ELISA test showed that among all sorghum genotypes, lines 6 and 10 had the least and the highest amount of total fumonisins respectively. The results of this experiment demonstrated that fumonisin production level in sorghum kernels was less than $5 \mu \mathrm{gg}^{-1}$ at all locations and Sari was the best location for fumonisin production on sorghum kernels in comparison with the other regions.

Rahjoo and Zamani [42]] evaluated the susceptibility of 10 lines of maize to Fusarium ear rot and fumonisins production by inoculating maize ears using the mixture of some virulent Fusarium verticillioides strains in two different inoculation methods. The evaluation was done on ears using Disease Severity Index (\%DS) 
4-5 weeks after inoculation. All infected ears were evaluated by ELISA kits (AgraQuant; Romer Labs, Austria) for their total fumonisins production at the physiological maturing stage. Lines B73 $\mathrm{cms}$ and $\mathrm{K} 74 / 1$ with $2.9 \mu \mathrm{gg}^{-1}$ and $72.2 \mu \mathrm{gg}^{-1}$ had the least and highest amount of total fumonisins respectively. The results of this experiment demonstrated that the Tip Injection method was better than Nail Punch based on the observed disease severity and measured fumonisins accumulation on inoculated ears.

To determine levels of total fumonisins production of some Fusarium isolates recovered from infected ears of maize with different geographic origins, a total of 37 isolates (37 F. verticillioides and one F. thapsinum) were evaluated by ELISA test Rahjoo et al. [39]. 14 out of 37 isolates were selected. Fumonisin analysis of F. verticillioides by ELISA test showed different levels of total fumonisins B production by grouping the isolates into three distinct levels as high $\left(>500 \mu \mathrm{gg}^{-1}\right)$, moderate $\left(100-500 \mu g^{-1}\right)$ and low $\left(<100 \mu \mathrm{gg}^{-1}\right)$ fumonisin producers. F. thapsinum produced a low amount of fumonisins. There was no clear correlation between fumonisin production levels of isolates and their geographic origins.

Rahjoo and Feizbakhsh [43] determined total fumonisins and AFs production on different grain sorghum at Karaj and Gorgan stations in 2015. The results of fumonisins analysis obtained from ELISA test showed that total fumonisins amount of sorghum genotypes ranged from 0.75 to $3.31 \mu \mathrm{gg}^{-1}$, and from 1 to $60 \mu \mathrm{gg}^{-1}$ in Karaj and Gorgan respectively, suggesting that fumonisin occurrence is probably related to humid conditions in the north of Iran. Total AFs amount of sorghum genotypes ranged from 2 to $24.23 \mathrm{ngg}^{-1}$, and from 2.7 to $80 \mathrm{ngg}^{-1}$ in Karaj and Gorgan respectively, indicating that AFs are also produced more under humid conditions in the north of Iran.

The first report of natural DON contamination of maize from Iran was reported in 2010 that Sixty maize samples were collected from different locations of Golestan and Moqan areas. DON contamination was found in $76.7 \%$ of samples in the range of 54.4$518.4 \mathrm{ngg}^{-1}$ [29]. To determine the possibility of DON contamination of maize in maize-wheat rotation crop, Moqhan region with about 10000 hectares' maize cultivation was selected for sampling. To study the potential of DON production of the isolates, ten isolates (two of each species) were selected as the representative of each species. Fusarium species and their frequency of isolation were F. verticillioides, 47.65\%; F. proliferatum, 33.873\%, 15.33\%; F. nygamai, $4.86 \%$; F. oxysporum, $3.06 \%$. DON was detected in $45 \%$ of the samples. The range of contamination was $35.4-542.55 \mathrm{ngg}^{-1}$. The mean of contamination was $165.7 \mathrm{ngg}^{-1}$ that was less than the maximum rate limit of DON which was recommended in the world $\left(1 \mu \mathrm{gg}^{-1}\right)$ [23]. Also, Mirabolfathy [30] reported DON contamination of the maize samples of Fars and Khuzestan provinces were $5 \%$ and $2 \%$ respectively and the amount of contamination was 21.3 - 97.4 (63.99) ngg $^{-1} .55 \%$ percent of maize samples collected from Ardabil province were contaminated with DON ranging from 35.4-542.55 (165.7) ng/g.

\section{Mycotoxins in dried fruits and nuts}

AFs contamination of nuts and dried fruits has remained as an important problem reported from different countries. Surveys have been conducted to measure AFs contamination level in nuts and dried fruits in Iran despite the incidence of AFs contamination in tree nuts is low, AFs levels can be variable and high levels and can develop in a small percentage of harvested nuts. For example, it has been estimated that only 1 in 28250 walnuts, 1 in 26500 almonds, and 1 in 25000 pistachios.

Iran National Standard Organization (INSO) has set the maximum limit for OTA and AFs levels in dried fruits and nuts. 10ngg-1 for OTA in raisin, date, and fig, $5 \mathrm{ngg}^{-1}$ for AFB1 and $15 \mathrm{ngg}^{-1}$ for total AFs [4].

\section{Pistachio}

AF contamination of pistachio nuts is undoubtedly an important economic problem for Iran. The European Union's banned pistachio imports from Iran because of high levels of AFs in 1997. The existence of AFs and fungal producing AFs in pistachio were reported from Iran in 1970. Since then many researches was conducted to find the fungal species produced AFs, it was reported that up to 13 species were isolated from pistachio kernels and shells collected from the orchard. The probability of contamination incidence in orchard, processing stages, at storage and shipment, were studied [31]. The results showed that the most important stage is during the washing time at processing stage while pistachio nuts contaminate with fungal spores and one of the most important stage which suggesting to play an important role for AFs contamination of pistachio nuts is orchards while the pistachio nuts are being developed and fungal contaminations are initiated through early split pistachio nuts. Another project was implemented for 5 years (205-2010) in which pistachio nuts were collected from the different storages and terminals of pistachio producing areas of Kerman province including Rafsanjan, Zarand, Kerman, Noogh, and Sirjan. 122 isolates of A. flavus collected using suspension of surfactant contamination of pistachio nuts. To detect fungal contamination at different parts of pistachio nuts, 51 samples of shells and kernels collected from 51 different storages were disinfected using hypochlorite $0.5 \%$ and cultured on artificial media separately. $86 \%$ of the samples were contaminated to A. flavus in a range of $0-100 \%$. Due to AFs contamination, $32 \%$ of the samples had AFs more than $2 \mathrm{ngg}^{-1}$ and $68 \%$ less than $2 \mathrm{ngg}^{-1}$. Morphological and several physiological characters of the collected A. flavus isolates were studied and grouped based on common characters. Representative isolates of each group selected, cultured on rice medium and evaluated toxicologically using TLC scanner method. $27 \%$ of isolates produced sclrotia. All the isolates which studied toxicologically produced AFs B1 with an average rate of $11 \pm 0.52 \mu \mathrm{gg}^{-1}$ and aflatoxin $\mathrm{B} 2$ with average rate $6.2 \pm 0.41 \mu \mathrm{gg}^{-1}$. No isolate produced aflatoxin G1 or G2. A criterion morphological character related to AFs production was not found in studied isolates [31]. 


\section{Hazelnut}

Approximately 12500 hectares of hazelnut trees were grown in Guilan province in the North of Iran. AFs contamination of hazelnut is an important exportation problem for the main producing countries. Mirabolfathy et al. [7,11] reported the result of their research project was conducted to clear the contamination risk of AFs and AFs -producing fungi in the hazelnut produced in Guilan mainly consumed locally. In this research project during two consequences years' AFs producing Aspergillus species were monitored in hazelnut samples collected from Eshkevarat and Deilaman dry land areas in Guilan province at harvest time, after two months storing at local storages and after processing at the local processing sites. AFs contaminations of the samples were estimated using HPLC+IAC. Despite the high occurrence frequency of AFs -producing fungi (45\%), the contamination level of AFs producing fungi and AFs were low (1-1.7\% and 0.1-0.4ngg ${ }^{-1}$ respectively) in hazelnuts samples collected at harvest time samples. The population density of A. flavus and A. parasiticus increased to $2.9 \%$ in hazelnut samples after 3 months of storing at local storages but due to closing the shells, AF contamination did not occur, and AFs were not found in the storage's samples. High levels of AFs (54n $\mathrm{g}^{-1}$ ) were determined and A. flavus and A. parasiticus were found more frequently (55\%) after processing stages including soaking for 48 hours at contaminated sites. Based on the results obtained from this project it is recommended that the traditional processing sites must be improved using HACCP principals.

\section{Fig}

Approximately 23000 hectares of fig orchards including cultivated and wild fig trees (Ficus caraca L.) in Fars province (Stahban dryland area) provide 23000 tons of dried figs produced in Iran. During recent years' exportation of dried figs encountered a problem due to its AFs contamination which was reported informally. Status of AFs contamination and the mechanisms of fig infection by $A$. flavus and $A$. parasiticus were required to reduce the risk of fig contamination. Mirabolfathy et al. [31] carried out a two year's research project and monitored AFs -producing - Aspergillus species in the samples of two cultivars of Capri fig (Ficus caricacv, Capri) samples, including Danesefid and Pouzdonbali, collected from Estahban and EiJ areas respectively in Jun. The edible fig samples collected from Calimyrna trees (Ficus carica cv, Calimyrina) located at eastern (Kezman) and western part (Tirvanjan) of Estahbanat dry land areas during harvest time, before processing, during processing stages, and after packaging. AFs contaminations of the samples were determined using HPLC+ IAC. A. flavus and A. parasiticus were not found in all the Capri fig samples for two years. A. flavus was found in $20 \%$ of the edible figs during the first year, the mean of contamination was $7.5 \%$ among the contaminated samples. During the second year, fungal contamination was not found among the edible figs collected from Calimyrna trees at harvest time, but it was found in $20 \%$ of samples were collected after harvest time, the mean of contamination in contaminated samples was $5 \%$. Aflatoxin B1 was the only AFs traced (at a level of $1 \mathrm{ngg}^{-1}$ ) in thirty- five percent of edible figs which were collected after harvest time and one sample of each before processing and after processing stages as well. A. flavus and AFs were not detected in a processed sample in the first year. During the second year, AFs were not detected in edible figs.

\section{Almond}

Mirabolfathy et al. [32] conducted a research project to clear the contamination risk of AFs and AFs producing fungi in almond produced in Iran. Approximately 185294 hectares of almond trees were grown in Fars, Khorasan, Chaharmahal Bakhtiari, Esfahan, Zanjan, Lorestan and Markazi provinces located in different geographical areas of Iran. In this research project AFs -producing Aspergillus species were monitored in almond samples collected from Fars, Khorasan, Chaharmahal Bakhtiari, Esfahan, Zanjan, Lorestan and Markazi provinces after harvesting. The natural contamination population density of A. flavus and A. parasiticus in almond samples was 10-80 percent. Despite the low occurrence frequency of AFs producing fungi in samples, there is a potential of AFs production in a favorable environmental condition to grow the fungal agents in some storages. High levels of AFs (376ngg-1) were determined in one sample collected from Arak area which was also contaminated to a high level of AFs -producing fungal spores.

\section{Raisin}

Ochratoxin (OTA) is a mycotoxin which contaminates different plant products, including cereals, coffee, and dried grape. Known for its nephrotoxic effects, OTA can also impact the liver. A major renal disease of swine known as porcine nephropathy occurs in some European countries and is associated with consumption of OTA -contaminated barley.

The presence of OTA in raisin is an important barrier to raisin export. The grape vine is cultured through 315000 hectares of Fars, Khorasan, Zanjan, Qazvin, Hamedan and Azarbaijan provinces. During 2010- 2012 a research was carried out on the monitoring of OTA and OTA - producing fungi in raisin of Khorasan, Azarbaijan, Qazvin and Zanjan provinces [7]. 43 samples collected from different processing stages of raisin including storages, and factories. To study the raisin fungal contamination two methods of plating: surface disinfected raisin and washing of raisin surface (suspension) on artificial media were used. 14 isolates selected as representative were cultured on PDB medium and OTA produced by each isolate was evaluated using HPLC- IAC method. All raisin samples were contaminated by Aspergillus section Nigri species. The mean of the assumed OTA producing -fungi contamination of raisin samples was $56.31(8-100 \%)$ but it varied depending on the processing method, raisin variety, and location. Twenty percent of the isolates belonged to A. carbonarus species and the rest to $A$. niger. $71 \%$ of tested $\mathrm{A}$. carbonarius isolates produced ochratoxin between $0.78-108.8 \mathrm{ngg}^{-1}$. Ochratoxin A was found in five samples out of $44(11 \%)$ raisin samples at the contamination levels of 0.4 to $100 \mathrm{ngg}^{-1}$. The highest contamination level was found in injured, non-edible raisin. In another study 10 raisin samples were analyzed by DLLME method, that one sample was contaminated at $6.48 \mathrm{ng} / \mathrm{g}$ [33]. 


\section{Mycotoxins in dairy products}

The occurrence of AFM1 in milk and milk products is a public health concern because the International Agency for Research on Cancer has classified it in Group 2, a probable human carcinogen with a high risk of hepatotoxicity and mutagenicity [34]. For this reason, milk and dairy products must be inspected and controlled continuously for AFM1 contamination and animal feeds should be checked regularly for AFB1 and storage conditions of feeds must be taken under strict control. Kazemi Darsanaki et al. [34] reviewed recent studies in aflatoxin M1 contamination in milk and milk products like yogurt and cheese in Iran and reported that the incidence of positive cheese samples for AFM1 seems to be widely variable. The maximum limit for AFM1 in milk and dairy products is ranged between 0.05 to $0.2 \mathrm{ng} / \mathrm{g}$ [4].

AFM1 is a hydroxylated metabolite of the carcinogen AFB1 and may be found in the milk of lactating animals that have ingested feedstuffs contaminated with AFB1. Rahimi et al., (2010a) examined 100 dairy product samples consisting of pasteurized milk (50 samples), and white cheese (50 samples), collected from retail markets during August 2009 to February 2010 in Ahvaz. Analytical results showed that $91(91 \%)$ samples ranging from 14 to $209 \mathrm{ngl}^{-1}$, consisted of $44(88 \%)$ pasteurized milk samples (range: $11-94 \mathrm{ng} / \mathrm{l}$ ) and 47 (94\%) white cheese samples (range: 22-209ngl-1 ) were contaminated with AFM1. Considering the US FDA and Iranian national standard limits for AFM1 in milk $\left(500 \mathrm{ngl}^{-1}\right.$ or $\left.0.05 \mathrm{ngg}^{-1}\right)$, none of the samples had levels above the maximum tolerance limit. However, the concentration levels of AFM1 in $3(6 \%)$ and $11(22 \%)$ samples of pasteurized and white cheese were higher than the European Commission limit, respectively. AFM1 contents of white cheese samples were higher than those of pasteurized milk samples. No significant differences in the concentration of AFM1 were observed for pasteurized milk samples taken in different seasons in Ahvaz. However, significantly higher concentrations of AFM1 $(\mathrm{P}<0.05)$ were found in white cheese samples taken in cold seasons. Although the concentration of AFM1 in none of the examined samples exceeded the Iran regulation, by attention to high consumption and significance of milk and its products in Iranian dietary, survey and control of milk and its products for a measure of AFM1 continuously has a high significance. Mohsenzadeh et al. [35] evaluated AFM1 contamination in raw milk samples in Mashhad, northeast of Iran by ELISA. For this purpose, two hundred raw milk samples were obtained from dairy plants of Mashhad city of Iran. AFM1 was found in 76\% of the raw milk samples by an average concentration of $0.061 \pm 0.004 \mu \mathrm{gkg}^{-1}$. The range of contamination levels varied among between months. The highest mean concentration of AFM1 in raw milk samples was registered in February samples $\left(0.225 \mathrm{ngg}^{-1}\right)$ and the lowest mean concentration registered in August samples (0.0058ngg ${ }^{-1}$ ). The statistical evaluation showed that there were significant differences $(\mathrm{P}<0.05)$ between the concentrations of AFM1 of raw milk samples taken in May and August with November and February. The concentrations of AFM1 in 36.5\% of the samples were higher than the maximum tolerance limit accepted by the European
Union/Codex Alimentarius Commission. It was therefore concluded that the levels of AFM1 in milk samples consumed in Mashhad, Iran was high and appear to be a serious public health problem now. Riahi Zanjani et al. [36] evaluated AFM1 contamination in 45 samples of raw milk was collected randomly from Fariman, Iran. AFM1 was detected in all milk samples. The mean concentration of AFM1 was $11.61 \pm 0.72 \mathrm{ng} / \mathrm{L}$ with a range of $6.3-23.3 \mathrm{ng} / \mathrm{L}$. None of the samples had levels exceeding the maximum tolerance limit (50ng/L) accepted by the European Union. The results showed that AFM1 contamination of milk is not a concern in this city. However, dairy cattle feed samples of various livestock must be regularly checked for AFs and the storage conditions of feeds should be strictly controlled.

\section{Mycotoxins in herbal drug products}

A study was carried out to determine the percentage incidences of mycoflora associated with the root samples, occurrence, and level of AFs in fresh and marketed drug roots. Rashidi et al. [37] selected five root herbal drugs to belong to Dashmoola for their investigation. Qualitative and quantitative estimation of AFs $(\mathrm{B} 1, \mathrm{~B} 2, \mathrm{G} 1, \mathrm{G} 2)$ was determined by the BGFY test and HPTLC method. The result indicated the percentage incidence of mycoflora especially different species of Aspergillus and the qualitative and quantitative estimation of AFs in some drug samples of fresh and most of the drugs samples from the market were contaminated with AFs especially AFB1 and AFB2. The results indicate that although the natural occurrence of AF in herbal medicines analyzed in this study was less and it was below of tolerance level $\left(20 \mu \mathrm{gkg}^{-1}\right)$ fixed by the World Health Organization but the contamination may be significant due to their frequent and prolonged consumption.

\section{Control of mycotoxins}

Mycotoxins are secondary metabolites of filamentous fungi that occur naturally in food and feed. There are limited investigations have been studied to decrease or eliminate the mycotoxins in foods and plant products in Iran. Some of them have been reported to date involve the use of microorganisms and chemical compounds to reduce the major mycotoxins.

Essential oils (EOs) effect on mycotoxins was studied in many research $[38,39]$. In a survey, inhibitory growth effects of Thymus vulgaris, Satureja hortensis, Anethum graveolens, Mentha sativa and Capsicum annum essential oils were studied on F graminearum and ZEA production [30]. The results showed that all the essential oils reduced ZEA production by $87-93 \%$, the most (93\%) and least $(87 \%)$ reduction of ZEA production were observed in C. annum (5000 $\left.\mu \mathrm{gg}^{-1}\right)$ and S. hortensis $\left(150 \mu \mathrm{gg}^{-1}\right)$ treatments, respectively. Lahoji [40] studied the effects of Zataria multiflora and S. hortensis essential oils, and thymol and carvacrol which are two main components of these essential oils on the growth of $10 \mathrm{~F}$. graminearum isolates and reduction of DON. Z. multiflora, S. hortensis, thymol, and carvacrol decreased $84,89.1,95$ and $86.6 \%$ of DON respectively [40]. The ability of Thymus daenensis, Satureja khozistanica, and Satureja macrosiphonia essential oils was investigated for reducing A. flavus growth and it's AFB1-content in the 
liquid culture, and results showed that T. daenensis at a concentration of $2000 \mathrm{mg} / \mathrm{l}$ reduced of $97 \%$ AFB1 (Goran et al. 2013).

Mirabolfathy et al. [7] studied the role of chemical control for reducing the fungi and AFs contaminations at the terminal using ozone. The objective of this study was to evaluate the capability of electrochemically produced ozone to degrade AFs and controlling AFs producing fungus in artificially contaminated pistachio nuts with an aflatoxicogenic isolate of $A$. parasiticus. The results showed that $A$. parasiticus population density of ozone treated pistachio nuts was decreased $90-92 \%$ and AFs contaminations of the treated pistachio nuts decreased $45-74 \%$.

One of the most important stage which suggesting playing an important role for AFs contamination of pistachio nuts is in orchards while the pistachio nuts are being developed and fungal contaminations are initiated through early split pistachio nuts. To study the role of chemical control for reducing the AFs contamination of pistachio nuts, an experiment was conducted in a completely randomized blocked design with 10 treatments and 4 replications in two successive years (2005-2006) in two regions, Koshkooie et al. in Kerman province [31]. Fungicides were including Bordu mixture $(1 \%)$ in winter, cupric chloride oxide $(1 \%)$ in spring, captan $\left(3 \mu g^{-1}\right)$ and Iprodione-Carbendazim (Rovral TS) $\left(2 \mu \mathrm{gg}^{-1}\right)$, two times at interval one month in summer. The results showed that the AFs contamination levels of pistachio nuts in the experimental orchards were less than $2 \mathrm{ngg}^{-1}$. So, in which range the effect of fungicides could not be compared, but the frequency of incidence reduced in Rovral TS treatments, while AFS producing fungi were considerably decreased in pistachio nuts at harvest time in fungicide treatments. Rovral TS decreased $A$. flavus and $A$. parasiticus contamination levels $50-70 \%$ compared with using other fungicides.

Several microorganisms have been reported to bind or degrade AFs in foods and feeds. During a survey was carried out by Chegini et al. [41], four isolates of Trichoderma were studied for their ability to reducing AFB1 produced by A. flavus on pistachio nuts. Two Trichoderma species T. harzianum and T. longibrachiatum were tested against $A$. flavus on pistachio nuts. Results showed that all four isolates of Trichoderma have a meaningful difference in reducing AFB1 production by A. flavus in pistachio nut as compared with control. Specific lactic acid bacterial strains remove toxins from media. Farzaneh et al. [42] evaluated the potential of the four Bacillus subtilis strains, isolated from pistachio nuts for AFB1 reduction. The results showed that one strain could perfectly inhibit the growth of A. flavus and production of AFB1 in the liquid culture. During recent decades' lactic acid bacteria have been used to prepare and improve storage of food and for ensiling of different crops for animal feed. The binding of AFB1 of the contaminated solution by 2 strains of lactic acid bacteria was studied by khanaferi, and the selected strains of Lactobacillus acidophilus (PTCC 1643) and L. plantarum (PTCC 1058) Bacteria were added to the spiked solution of $50 \mathrm{ngg}^{-1}$. The L. plantarum strain removed $45 \%$ of AFB1 from solution for 1 hour and $100 \%$ of it during 90 hours of incubation [43]. Saccharomyces cerevisiae is one of the major microorganisms widely used in food fermentation, and the ability of its strains to reduce the level of AFs has been reported. Karami-Osboo et al. [40] reported that different strains of S. cerevisiae reduced the AFs levels in a different rate and various duration times, and at the time 320 min the PTCC 5052 strain reduced the AF B1, B2, G1 and G2 levels to $11.2,13.9,8.0$ and 8.1\%, respectively; meanwhile, these results for the PTCC 5269 strain 9.5, 8.0, 2.3 and $16.2 \%$, respectively. Results suggested that different strains of $S$. cerevisiae had a different reduction rate on AFs. Moreover, the strains need to have enough time to absorb the maximum amounts of AFs [32].

Some physical treatment such as roasting, washing, and cooking was studied on the reduction of some mycotoxins in cereal and nut; these methods has reduced mycotoxin in food matrices [44-54].

\section{References}

1. Anonymous, 2018. Agricultural statistical report. Ministry of Jihad-e-Agriculture of the Islamic Republic of Iran.

2. Aliakbari Z, Mirabolfathy M, Aminian H, Karami-Osboo R (2011) Fusarium species and Deoxynivalenol in maize product of Moqan region. Appl Ent Phytopath 79(2): 1-18.

3. Babaei-Razdari H, Chaichi Nosrati A, Ghaemi N, Mehr Ara S (2014) An assessment of aflatoxin levels in wheat samples of 5 top provinces of Iran. Bulletin of Environment Pharmacology and Life Sciences 3(5): 237-242.'

4. Balazs E, Schepers JS (2007) The mycotoxin threat to world safety. International Journal of Food Microbiology 119: 1-2.

5. Beheshti HR, Asadi M (2014) Aflatoxins in animal feed in Iran. Food Addit Contam Part B Surveill 7(1): 40-42.

6. Bennett JW, Klich M (2003) Mycotoxins. Clin Microbiol Rev 16(3): 497516.

7. Castegnaro M, McGregor D (1998) Carcinogenic risk assessment of mycotoxins. Revue Med Vet 149(6): 671-678.

8. Chegini S, Behboudi K, Javan-Nikkhah M, Farzaneh M (2014) Inhibition of Aspergillus flavus and aflatoxin B1 reduction in Pistachio by Trichoderma strains. Biological Control of Pests and Plant Diseases 2(2).

9. Davari M, Safaie N, Darvishnia MR (2014) The occurrence of deoxynivalenol producing isolates of Fusarium graminearum species complex associated with the head blight of wheat in Moghan area. Journal of Crop Prptection 3(2): 113-123.

10. Del Ponte EM, Garda-Buffon J, Badiale-Furlong E (2012) Deoxynivalenol and nivalenol in commercial wheat grain related to Fusarium head blight epidemics in southern Brazil. Food Chem 132(2): 1087-1091.

11. European Commission (2006) Commission Regulation EC No. 401/ (2006) of $23^{\text {rd }}$ February 2006, laying down the methods of sampling and analysis for the official control of the levels of mycotoxins in foodstuffs. Official Journal of the European Union, EU publication, L 70/12.

12. Farzaneh M, Qi Shi Zhi, Ghassempour A, Sedaghat N, Ahmadzadeh M, et al. (2012) Aflatoxin B1 degradation by Bacillus subtilis UTBSP1 isolated from pistachio nuts of Iran. Food Control 23(1): 100-106.

13. Feizy J, Beheshti HR, Fahim NK, Janati SS, Davari G (2010) Survey of aflatoxins in rice from Iran using immunoaffinity column clean-up and HPLC with fluorescence detection. Food Addit Contam Part B Surveill 3(4): 263-267. 
14. Gelderblom WC, Jaskiewicz K, Marasas WF, Thiel PG, Horak RM, et al. (1988) Fumonisins - Novel Mycotoxins with cancer- promoting activity produced by Fusarium moniliforme. Appl Environ Microbiol 54(7): 1806-1811.

15. Ghiasian SA, Rezayat SM, Kord-Bacheh P, Maghsood AH, Yazdanpanah $\mathrm{H}$, et al. (2005) Fumonisin production by Fusarium species isolated from freshly harvested maize in Iran. Mycopathologia 159(1): 31-40.

16. Ghiasian SA, Maghsood AH, Yazdanpanah H, Shephard GS, Van Der Westhuizen L, et al. (2006) The incidence of Fusarium verticillioides and Levels of Fumonisins in Maize from Main Production Areas in Iran. J Agric Food Chem 54(16): 6118-6122.

17. Habib V, Mohammad G, Zeinal-Abedin B, Zahra M (2017) Ochratoxin a detection in rice samples in mazandaran province. Pharmacophore $8(6): 10-21$

18. Hoseiniyeh Faraahani SH, Mirabolfathy M, Rezaie Danesh Y, Karami Osboo R (2013) Effect of five essential oils on zearalenon production and growth of Fusarium graminearum. Applied Entomology and Phytopathology 80(1): 81-94.

19. Institute of Standards and Industrial Research of Iran. 2002. Food and feed mycotoxins: maximum tolerated level. 1st ed. ISIRI No. 5925. Tehran: ISIRI.

20. Kazemi Darsanaki, R, Doost Chakoosari M, Azizollahi Aliabadi M (2013) Aflatoxin M1 Contamination in Milk and Milk Products in Iran: A Review. Journal of Chemical Health Risks 3(3): 13-20.

21. Karami Osboo R, Mirabolfathy M (2008) Natural Zearalenone Contamination of Wheat from Golestan Province, Northern Iran. Iranian Journal of Plant Pathology 44: 60-65.

22. Karami-Osboo R, Maham M (2018) Pre-concentration and Extraction of Aflatoxins from Rice Using Air-Assisted Dispersive Liquid-Liquid Microextraction. Food Analytical Methods 11(10): 2816-2821.

23. Karami-Osboo R, Mirabolfathy M, Aliakbari F (2010) Natural deoxynivalenol contamination of maize produced in Golestan and Moqan areas in Iran. Journal of Agricultural Science and Technology 12(2): 233-239.

24. Karami-Osboo R, Mirabolfathy M, Kamran R, Shetab-Boushehri M, Sarkari S (2011) Aflatoxin B1 in maize harvested over 3 years in Iran. Food Control 23(1): 271-274

25. Karami-Osboo R, Miri R, Javidnia K, Kobarfard F, AliAbadi MH, et al (2015) A validated dispersive liquid-liquid microextraction method for extraction of ochratoxin A from raisin samples. J Food Sci Technol 52(4): 2440-2445.

26. Karami-Osboo R, Mirabolfathy M (2016) Effect of different strains of saccharomyces cerevisiae on the reduction of aflatoxin B1, B2, G1 and G2. Journal of food hygiene 6(32300258): 43-53.

27. Khanafari A, Soudi H, Miraboulfathi M, Osboo RK (2008) An in vitro Investigation of Aflatoxin B1 uptake by Lactobacillus plantarum. Pak J Biol Sci 10(15): 2553-2556

28. Lahooji A, Mirabolfathy M, Karami-Osboo R (2010) Effect of Zataria multiflora and Satureja hortensis essential oils, thymol and carvacrol on the growth of Fusarium gramineum isolates and deoxynivalenol production. Iranian Journal of Plant Pathology 46(1): 37-50.

29. Maham M, Kiarostami V, Waqif-Husain S, Karami-Osboo R, Mirabolfathy M (2013) Analysis of ochratoxin A in malt beverage samples using dispersive liquid-liquid microextraction coupled with liquid chromatography-fluorescence detection. Czech Journal of Food Sciences 31(5): 520-525.

30. Mohsenzadeh M, Dastour M, Aelami M, Asad Zadeh (2010) A study on the occurrence of aflatoxin M1 in raw milk samples in Mashhad, Northeast of Iran. Proceeding of International Mycotoxin Conference, MycoRed, Penang, Malaysia, pp. 199.
31. Mianabi S, Mirabolfathy M, M Ghayeb-Zamharir (2014) Molecular studies on Fusarium graminearum clade of the wheat crop at Ardabil province in Iran. Agricultural Biotechnology 13: 89-97

32. Mirabolfathy M, Karami Osboo R, Amini H (2006) Fumonisin B1 Contamination of Golestan Maize Product. Iranian. Journal of Plant Pathology 42(2): 359-374.

33. Mirabolfathy M, Karami-Osboo R (2013) Deoxynivalenol and DON producing Fusarium graminearum isolate in wheat and barley crop at north and northwest areas of Iran. Iran J Plant Path 48(4): 197-210.

34. Mirabolfathy M, Karami-Osboo R, Hosseinian L (2014) Ochratoxin A and ochratoxin - producing isolates of Aspergillus section Nigri in a raisin in Iran. Iran J Plant Path 50(1): 1-16.

35. Moradi Ghahderijani M, Mirabolfathy M (2007) The population density of Aspergillus flavus and Aspergillus niger groups at different kinds of pistachio processing terminals in Kerman province. Pajouhesh - va Sazandegi 20(4): 104- 110.

36. Mousavizadeh A, Peikar A, Hoseinian A, Ghaydie E, Pourmahmoudi A (2017) Determination of total aflatoxin in rice consumption in Yasuj, Iran. Biosci. Biotech Res Comm 1: 195-198.

37. Rahimi E, Rafei M, Nilchian Z, Hajmohamadi A, Reahi M (2010) Aflatoxin M1 in pasteurized milk and white cheese in Ahvaz, Iran. Proceeding of International Mycotoxin Conference, MycoRed, Penang, Malaysia, pp. 149.

38. Rahimi E, Kajbafi M, Jafarian M, Shakerian A, Hajian S, et al. (2010) Survey of ochratoxin A in rice from the retail market in Shahrekord, Iran. Proceeding of International Mycotoxin Conference, MycoRed, Penang, Malaysia, p. 203.

39. Rahjoo V, Shepherd GS, Klemsdal SS, Zad J, Javan NM, et al. (2008) Evaluation of fumonisin production for some Fusarium isolates recovered from corn ears in Iran. XII International Congress of Mycology, Istanbul, Turkey, p 51.

40. Rahjoo V, Parchamian M, Feizbakhsh MT, Zamani M (2011) Quantization of total fumonisins producing Fusarium verticillioides in some Maize and Sorghum genotypes by ELISA. Proc Nat Sci Matica Srpska Novi Sad, 121: 61-70.

41. Rahjoo V, Atabaki N (2014) Fumonisin detection on different genotypes of grain sorghum in Iran. The International Mycotoxin Conference, Beijing, China.

42. Rahjoo V, Zamani M (2014) Assessment of maize lines susceptibility to Fusarium ear rot and Fumonisin accumulation. The International Mycotoxin Conference, (Oral Presentation), Beijing, China.

43. Rahjoo V, Feizbakhsh MT (2016) Determination of total fumonisins and aflatoxins levels in some grain sorghum lines in Iran. Conference Abstracts. 38th Mycotoxin Workshop Berlin, Germany, pp 122.

44. Rasooli I, Owlia P (2005) Chemoprevention by thyme oils of Aspergillus parasiticus growth and aflatoxin production. Phytochemistry 66(24): 2851-2856.

45. Rastegar H, Shoeibi S, Yazdanpanah H, Amirahmadi M, Khaneghah AM, et al. (2017) Removal of aflatoxin B1 by roasting with lemon juice and/ or citric acid in contaminated pistachio nuts. Food control 71: 279-284.

46. Razzaghi-Abyaneh M, Shams-Ghahfarokhi M, Yoshinari T, Rezaee MB Jaimand K, et al. (2008) Inhibitory effects of Satureja hortensis L. essential oil on growth and aflatoxin production by Aspergillus parasiticus. Int J Food Microbiol 123(3): 228-233.

47. Rashidi M, Deokule SS (2013) Associated fungal and aflatoxins contamination in some fresh and market herbal drugs. Journal of Microbiology and Biotechnology Research 3(1): 2.

48. Rezaeian Doloei R, Rezaee S, Mirabolfathy M, Zamanizadeh H (2011) Molecular identification and detection of gene encoding deoxynivale- 
nol in Fusarium graminearum isolates, the causal agent of Fusarium head blight of wheat in Iran. Modern Science of Sustainable Agricultural Journal 7: 19-26.

49. Rezaeian DR, Rezaee S, Mirabolfathy M, Zamanizadeh H, Karami-Osboo R (2015) Determination of DON and NIV chemotypes of Fusarium graminearum on wheat in Iran. Applied Entomology Phytopathology 83: 1-11.

50. Riahi Zanjani B, Rahmani R, Rezayat Sorkhabadi SM, Aryan E, Oskouei Z, et al. (2015) A Survey on Aflatoxin M1 in Raw Milk of Fariman City, Khorasan Province, Iran. Jundishapur J Nat Pharm Prod 10(2): e20081.

51. Safara M, Zaini F, Hashemi S, Mahmoudi M, Khosravi A, et al. (2010) Aflatoxin detoxification in rice using citric acid. Iran J Public Health 39(2): 24-29.

52. Sani AM, Azizi EG, Salehi EA, Rahimi K (2014) Reduction of aflatoxin in rice by different cooking methods. Toxicol Ind Health 30(6): 546-550.

53. Shephard GS (2000) Liquid chromatographic method for fumonisins in maize. In: Trucksess MW, Pohland AE (Eds). Methods in molecular biology, Mycotoxin protocols, Humana Press, Totowa, NJ, USA, 157: 147-158.
54. Smith CW, Frederiksen RA (2000) Sorghum: origin, history, technology, and production, New York, NY John Wiley, and Sons, USA, pp. 824.

55. Tavakoli H, Rostami H, Zabihi A, Bitarafan Z (2014) A survey of aflatoxins, ochratoxin and Zearalenone Contamination in Imported and Iranian Rice in Iran. Agricultural Engineering Research Journal 4(3): 61-64.

56. Yazdanpanah H, Miraglia M, Calfapietra FR, Brera C, Rasekh HR (2001) Natural occurrence of mycotoxins in cereals from Mazandaran and Golestan provinces. Arch Iranian medicine 4(3): 107-114.

57. Yazdanpanah H, Mohammadi T, Abouhossain G, Cheraghali AM (2005) Effect of roasting on the degradation of aflatoxins in contaminated pistachio nuts. Food Chem Toxicol 43(7): 1135-1139.

58. Yazdanpanah H, Shephard GS, Marasas WF, van der Westhuizen L, Rahimian H (2006) Human dietary exposure to fumonisin B1 from Iranian maize harvested during 1998-2000. Mycopathologia 161(6): 395-401.

Your next submission with Juniper Publishers
will reach you the below assets
- Quality Editorial service
- Swift Peer Review
- Reprints availability
- E-prints Service
- Manuscript Podcast for convenient understanding
- Global attainment for your research
- Manuscript accessibility in different formats
( Pdf, E-pub, Full Text, Audio)
- Unceasing customer service
Track the below URL for one-step submission
https://juniperpublishers.com/online-submission.php

